Reprod. Nutr. Dévelop., 1986, 26 (2 B), 589-595.

\title{
Relations entre les sécrétions mammaires et les teneurs en nutriments énergétiques et azotés sanguins chez la chèvre au début de la lactation
}

\author{
D. SAUVANT, J. C. ROBERT $\left({ }^{*}\right)$, D. LEGENDRE, F. TERNOIS, J. HERVIEU, \\ M. DURON $(*)$
}

Station de Nutrition et alimentation (I.N.R.A.) Institut national agronomique Paris-Grignon, 16, rue Claude Bernard, 75231 Paris Cedex 05 (*) A.E.C., 03600 Commentry.

Summary. Relationships between udder secretion and plasma energy and protein contents at the onset of lactation in the dairy goat.

An experiment was performed on 29 dairy goats at the onset of lactation ; energy and protein deficits were maximal during the first week of lactation. This was confirmed for energy by the plasma contents of non-esterified fatty acids (NEFA), $\beta$-OH-butyrate and glucose. However, protein deficit was more critical during the second week, as shown by plasma urea, glycine and 3-CH3-histidine contents. At that time, nutritional status depended more on udder production than on intake level. Moreover, the nutritional parameters of body lipid and protein mobilization were positively correlated. Milk protein content was positively correlated with the nutritive balance and status of the goats.

\section{Introduction.}

Le démarrage de la lactation est associé chez la chèvre, comme chez les autres femelles productrices, à un déficit nutritif qui se résorbe au fur et à mesure de l'éloignement de la parturition. Cette période de déficit fait l'objet de recherches en raison de son importance pratique. Le travail décrit s'inscrit dans ce cadre, plus précisément il a pour objet de confirmer, dans le cas des chèvres, certaines relations observées chez d'autres espèces entre la sécrétion lactée et la teneur de certains nutriments plasmatiques connus pour traduire l'état nutritionnel énergétique ou azoté de l'animal.

\section{Matériel et méthodes.}

L'étude porte sur 29 chèvres de race alpine ou Saanen du troupeau expérimental de la Station I.N.R.A. de Nutrition et Alimentation de I'Institut National Agronomique Paris-Grignon. Les animaux reçoivent en ration de base du foin de luzerne en quantité limitée (500 g MS/animal/jour) et de l'ensilage de pulpe de betteraves surpressées distribué ad libitum (500 à $875 \mathrm{~g} \mathrm{MS}$ ingérée/animal/jour). 
L'alimentation concentrée est distribuée au prorata de l'évolution de la production laitière des animaux : $750 \mathrm{~g} / \mathrm{MS} /$ jour en moyenne pour les semaines 1, 2, 3 et $950 \mathrm{~g} \mathrm{MS} /$ jour au cours des 5 semaines suivantes. Les quantités de matière sèche ingérées des différents aliments et la production laitière sont enregistrées quotidiennement. Les mesures de poids vif et des taux butyreux et protéiques du lait sont effectuées de façon hebdomadaire pendant deux jours successifs. Les résultats des mesures de digestibilité in vivo de la matière organique et I'analyse des aliments permettent de calculer leur valeur nutritive énergétique et azotée la plus probable. Les données d'ingestion, de production et de valeur nutritive sont utilisées pour calculer les bilans énergétiques et azotés, exprimés en kcal lait et P.D.I. respectivement (I.N.R.A., 1978).

Au milieu des semaines $1,2,4,6$, des prélèvements de sang sont effectués le matin juste avant la distribution du premier repas. Les teneurs en acides aminés libres du sang sont dosés selon la méthode décrite par Pawlak et Pion (1968). Les teneurs du plasma en glucose, AGNE et B-OH-butyrate sont mesurées selon les méthodes couramment appliquées au laboratoire.

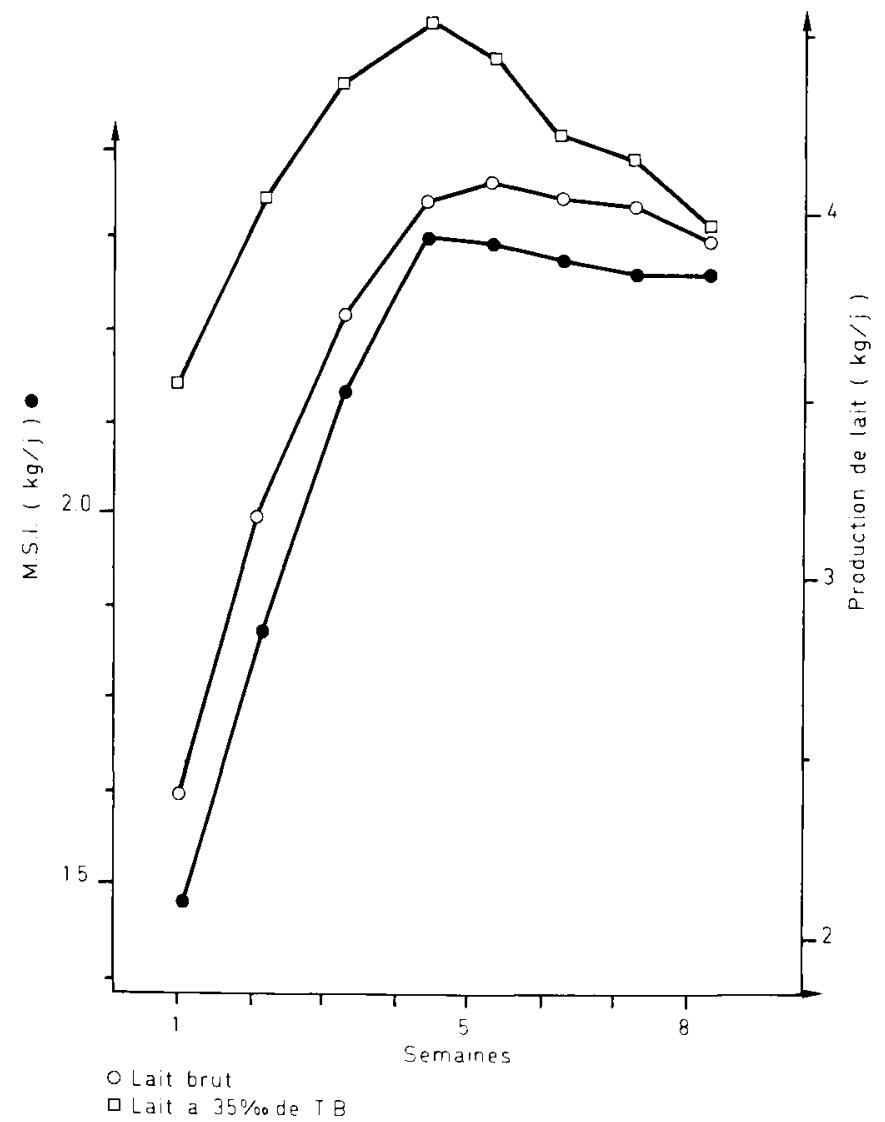

FIG. 1. - Evolution des quantités de matière sèche ingérées et de lait produites pendant les 2 premiers mois de lactation. 


\section{Résultats.}

Les figures 1 et 2 rapportent les évolutions, dans le temps, des quantités de matière sèche ingérées, de la production laitière et des taux butyreux et protéiques du lait. La figure 3 indique que la restauration de la valeur du bilan énergéti-

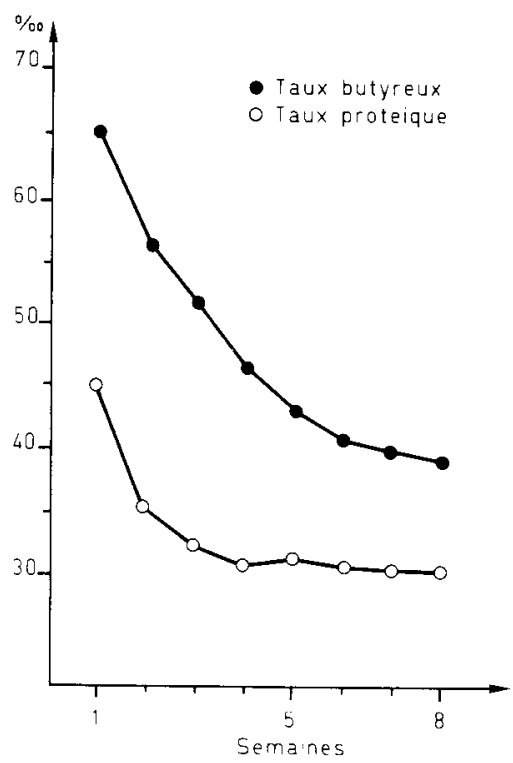

FIG. 2. - Evolutions des taux protéique et butyreux du lait.

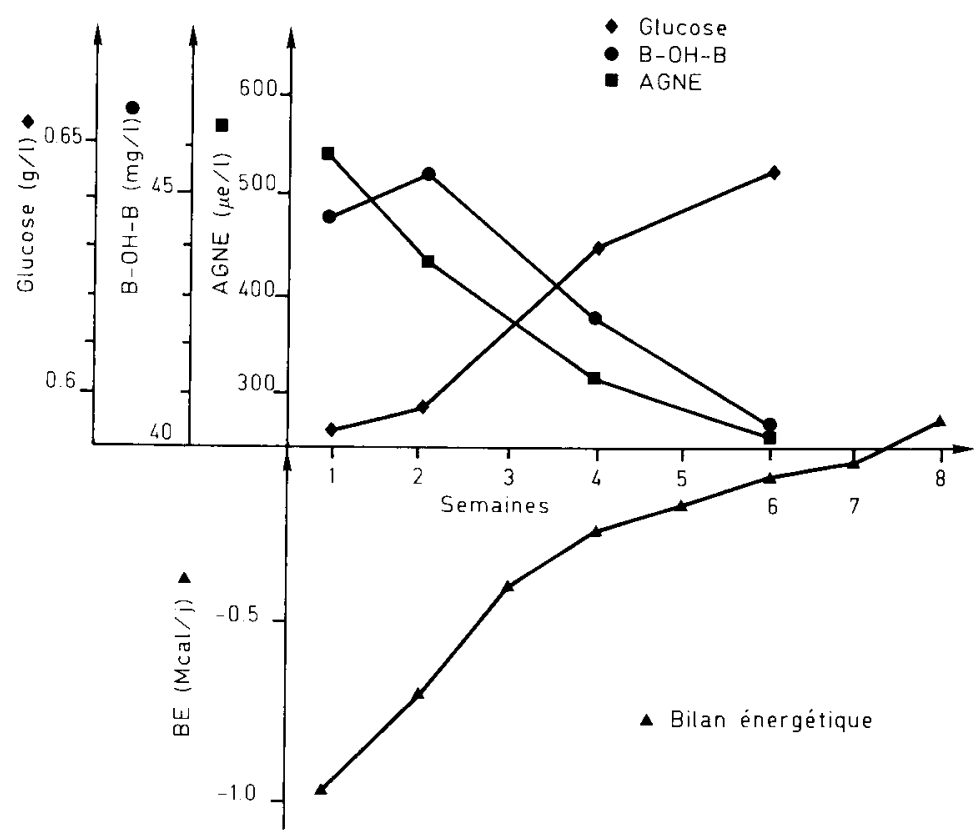

FIG. 3. - Evolution de l'état nutritionnel énergétique. 
que est associée, au niveau plasmatique, à un accroissement de la glycémie et une chute des teneurs en AGNE et B-OH-butyrate. La figure 4 montre que le bilan P.D.I. calculé devient positif à partir de la $3^{\mathrm{e}}$ semaine de lactation. L'urémie présente une valeur minimale en $2^{\ominus}$ semaine $(0,170 \mathrm{~g} / \mathrm{l})$ pour croître lentement ultérieurement. Les teneurs du sang en glycine et 3-méthyl-histidine sont maximales au cours de la seconde semaine de lactation. Les relations entre les différents paramètres mesurés sont plus marquées au cours de la seconde semaine comparée aux trois autres. Pour cette raison, l'interprétation des variations interindividuelles a été focalisée à cette période.

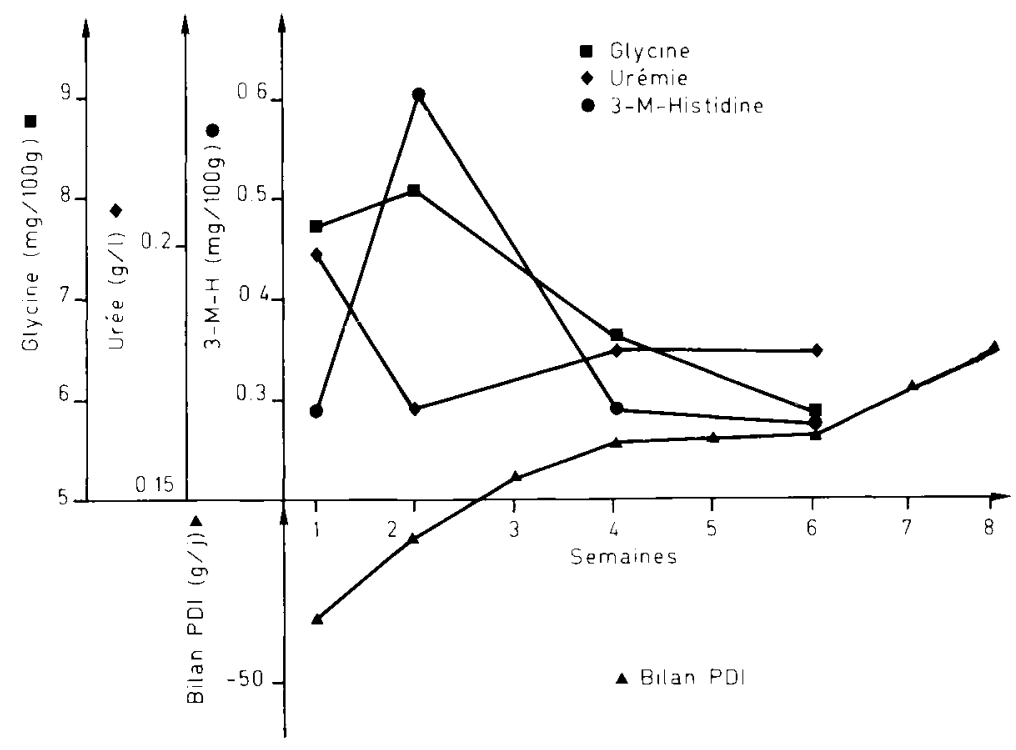

FIG. 4. - Evolution de l'état nutritionnel azoté.

En seconde semaine, les différences individuelles des bilans énergétiques et P.D.I. sont fortement corrélées $\left(0,98^{* *}\right)$. Elles sont, en outre, plus déterminées par les niveaux de production de lait brut $\left(-0,96^{* *}\right.$ et $-0,97^{* *}$ respectivement) que $d^{\prime}$ ingestion de matière sèche $\left(-0,35\right.$ et $-0,37^{*}$ respectivement). Les teneurs du plasma en glucose, AGNE et $\mathrm{B}-\mathrm{OH}$-butyrate ne sont pas liées aux variations de l'ingestion mais à celles de la production et des bilans nutritifs calculés (tabl. 1). A ce moment, la glycémie traduit mieux les variations des bilans nutritifs que les teneurs en $\mathrm{AGNE}$ et $\mathrm{B}-\mathrm{OH}$-butyrate. Les teneurs du sang en glycine, sérine et 3-méthyl-histidine sont significativement liées, de façon positive, avec le niveau de production et négative avec les bilans nutritifs calculés (tabl. 1). Les teneurs en valine et tyrosine présentent des relations inverses de celles qui viennent d'être décrites (tabl. 1).

L'urémie est comprise entre 0,05 et $0,47 \mathrm{~g} / \mathrm{l}$. Elle est positivement liée aux teneurs en acides aminés du cycle de l'urée : la citrulline $(0,29)$, l'ornithine $\left(0,55^{* *}\right)$, l'arginine $\left(0,63^{* *}\right)$, et à la valinémie $\left(0,44^{*}\right)$. Elle est par contre négativement liée à la teneur du sang en glycine $\left(-0,42^{*}\right)$. 
TABLEAU 1

Corrélations entre les principales caractéristiques zootechniques et certains nutriments sanguins en seconde semaine de lactation.

\begin{tabular}{|c|c|c|c|c|c|}
\hline & Moyenne & $\begin{array}{c}\text { Matière sèche } \\
\text { ingérée }\end{array}$ & $\begin{array}{l}\text { Production } \\
\text { de lait }\end{array}$ & $\begin{array}{c}\text { Bilan } \\
\text { énergétique }\end{array}$ & Bilan P.D.I. \\
\hline Moyenne & & $2,18 \mathrm{~kg} / \mathrm{j}$ & $3,24 \mathrm{~kg} / \mathrm{j}$ & - 854 kcal lait & $-20 \mathrm{~g} / \mathrm{j}$ \\
\hline$\left\{\begin{array}{l}\text { Glycémie } \\
\text { AGNE } \\
\text { B-OH-Butyrate }\end{array}\right.$ & $\begin{array}{c}0,60 \mathrm{mg} / \mathrm{l} \\
435 \mu \mathrm{\mu e} / \mathrm{l} \\
45,4 \mathrm{mg} / \mathrm{l}\end{array}$ & $\begin{array}{r}-0,27 \\
-0,02 \\
0,09\end{array}$ & $\begin{array}{c}-0,64^{* *} \\
0,37^{*} \\
0,43^{*}\end{array}$ & $\begin{array}{c}0,68^{* *} \\
-0,43^{*} \\
-0,37^{*}\end{array}$ & $\begin{aligned} & 0,67^{* *} \\
- & 0,45^{*} \\
- & 0,40^{*}\end{aligned}$ \\
\hline$\left\{\begin{array}{l}\text { Glycine } \\
\text { Sérine } \\
\text { Valine } \\
\text { Tyrosine } \\
\text { 3-méthyl-histidine }\end{array}\right.$ & $\begin{array}{l}8,21 \mathrm{mg} / 100 \mathrm{~g} \\
1,42 \mathrm{mg} / 100 \mathrm{~g} \\
2,01 \mathrm{mg} / 100 \mathrm{~g} \\
0,93 \mathrm{mg} / 100 \mathrm{~g} \\
0,60 \mathrm{mg} / 100 \mathrm{~g}\end{array}$ & $\begin{array}{r}-0,09 \\
0,19 \\
-0,24 \\
-0,11 \\
0,20\end{array}$ & $\begin{aligned} & 0,58^{* *} \\
& 0,57^{* *} \\
&-0,59^{* *} \\
&-0,40^{*} \\
& 0,68^{* *}\end{aligned}$ & $\begin{array}{rl}- & 0,61^{* *} \\
-0,56^{* *} & 0,61^{* *} \\
& 0,40^{*} \\
- & 0,71^{*}\end{array}$ & $\begin{array}{l}-0,64^{* *} \\
-0,59^{* *} \\
0,65^{* *} \\
0,43^{*} \\
-0,73^{* *}\end{array}$ \\
\hline
\end{tabular}

niveau de signification : $5 \%{ }^{*}, 1 \%{ }^{* *}$.

Des corrélations positives associent les nutriments, images de la lipomobilisation, $\mathrm{R}$ (AGNE, B-OH-butyrate) $=0,63^{* *}$ et de la mobilisation protéique, $\mathrm{R}$ (glycine, 3- $\mathrm{CH}_{3}$-histidine) $=0,68^{* *}$. Sur la base de ces deux relations, il est possible d'établir deux indices à caractères plus synthétiques :

un indice de lipomobilisation : IL $=\left[\right.$ AGNE $\left.\left({ }^{*}\right)+B-O H-B(*)\right] \times \frac{205,1}{435,5}$

un indice de mobilisation protéique : IP $=[$ (glycine $)+10\left(3-\mathrm{CH}_{3}\right.$-histidine $\left.)\right] / 2$ Les modes de construction de ces indices permettent de les exprimer respectivement en teneurs en AGNE et en glycine. Les valeurs de ces deux indices sont positivement et significativement corrélés $\left(0,70^{* *}\right)$.

Les taux protéiques du lait sont liés positivement aux valeurs des bilans énergétique et azoté $\left(0,49^{* *}\right.$ et $\left.0,54^{* *}\right)$, à la teneur en valine du sang $\left(0,42^{*}\right)$ et négativement à la production de lait $\left(-0,61^{* *}\right)$, à la teneur en $3-\mathrm{CH}_{3}$-histidine du sang $\left(-0,42^{*}\right)$ et à celle en $\mathrm{B}-\mathrm{OH}$-butyrate du plasma $\left(-0,48^{* *}\right)$. Les valeurs du taux butyreux du lait ne sont, par contre, pas corrélées aux différentes caractéristiques liées aux variations du taux protéique.

\section{Discussion et conclusion.}

L'évolution des niveaux de sécrétion et d'ingestion ainsi que des valeurs des bilans nutritifs calculés est conforme à ce qui est généralement observé chez les ruminants, en particulier les chèvres, au démarrage de la lactation. Sur la base des mesures effectuées, il apparaît que le déficit du bilan énergétique et la lipomobilisation sont maxima en première semaine de lactation alors que l'état nutritionnel azoté paraît être plus critique pendant la seconde semaine. En effet, à ce moment, d'une part l'urémie est minimale, et la valeur observée traduit un état de

(') Variables normées réduites. 
subcarence azotée et d'autre part, les teneurs en glycine et surtout en $3-\mathrm{CH}_{3^{-}}$ histidine sont maximales. Ces deux caractéristiques sont connues comme indicatrices de la mobilisation des protéines corporelles (Champredon et Pion, 1972, Joassart, 1976).

En seconde semaine, l'état nutritionnel des animaux est nettement plus dépendant des variations des niveaux des sécrétions mammaires que de celles des niveaux d'ingestion. Ces relations traduisent la priorité de la sécrétion lactée vis-àvis des métabolismes de l'organisme (téléophorèse) et la relative inadaptation de la capacité d'ingestion à l'accroissement des dépenses de production.

Les 29 animaux considérés produisent, en moyenne, plus de lait 13,24 vs $2,72 \mathrm{~kg} / \mathrm{j}$ ) que la population de 33 animaux étudiés au même moment dans une autre expérimentation (Sauvant et Robert, 1983). Cependant, les principales relations entre les caractéristiques mesurées en seconde semaine de production sont comparables entre ces deux expérimentations et ont déjà fait l'objet d'une discussion.

Le travail présenté fournit certains résultats complémentaires : la teneur du sang en 3- $\mathrm{CH}_{3}$-histidine non mesurée en 1983 se révèle être un meilleur indicateur du bilan azoté que les teneurs en glycine ou sérine. D'autre part, l'urémie, en moyenne plus faible que dans la référence précitée $(0,17$ vs $0,42 \mathrm{~g} / \mathrm{l})$, traduit la réduction globale de l'apport azoté et, dans ce cas, ses variations sont négativement liées au niveau de mobilisation protéique, relation qui n'était pas apparente dans le travail de Sauvant et Robert (1983). Les teneurs en tyrosine et surtout valine sont les plus négativement liées au niveau des sécrétions mammaires et pourraient, de ce fait, être considérées comme facteur limitant primaire. Nous avions fait une observation comparable pour la valine au même stade de production (Sauvant et Robert, 1983) alors qu'en $4^{\text {e }}$ semaine l'histidine et la phénylalanine étaient apparues comme les acides aminés les plus limitants (Sauvant et Robert, 1986).

Les relations associant, en seconde semaine les paramètres sanguins, images des phénomènes de mobilisation protéique et lipidique, confirment la corrélation entre les valeurs des bilans calculés et traduit vraisemblablement une part de régulation commune entre les processus de mobilisation ; il serait intéressant d'évaluer dans quelles mesures l'ensemble des métabolismes (minéraux...) est concerné par ce phénomène. En outre, la corrélation significative associant les indices de mobilisation indique que ces indices ne présentent qu'environ $50 \%$ de variance commune ; il n'est pas possible à travers nos résultats de savoir si cette valeur traduit les incertitudes attachées aux différentes mesures ou bien si elle traduit le fait que les animaux présentent une aptitude à mobiliser avec des degrés d'intensité différentiels leurs réserves protéiques et lipidiques.

Le taux protéique du lait est dans ce travail significativement plus faible chez les animaux présentant un état nutritionnel plus déficitaire. Nous avons confirmé cette tendance sur un nombre plus important (89) de chèvres considérées au même stade physiologique (résultats non publiés). Sur les vaches laitières, il est connu que le déficit énergétique est associé à une chute du taux protéique du lait (Journet et Rémond, 1978). Notre observation est peut être à rapprocher de cette tendance ; cependant, sachant que le taux protéique du lait varie selon un poly- 
morphisme génétique des caséines $\alpha_{\mathrm{s}}$ (Boulanger et al., 1984 et non publié), il conviendrait de pouvoir préciser dans quelle mesure la relation que nous observons pourrait être expliquée par des différences génotypiques.

11 Réunion du groupe Développement I.N.R.A. Montpellier, 22-24 mai 1985.

\section{Références}

BOULANGer A., GROSClAUde F., MAHÉ M., 1984. Polymorphisme des caséines $\alpha_{\mathrm{s} 1}$ et $\alpha_{\mathrm{s} 2}$ de la chèvre (Capra hircus). Génét. Sél. Evol., 16, 157-176.

CHAMPREDON C., PION R., 1972. C.R. Soc. Biol., 166, 378-381.

INRA, 1978. L'alimentation des ruminants, ed. I.N.R.A. Publications.

JOASSART J. M., 1976. Z. Tierphysiol. Tierernähr und Futtermittelk., 36, 205-210.

PAWLAK M., PION R., 1968. Influence de la supplémentation des protéines de blé par des doses croissantes de lysine sur la teneur en acides aminés libres du sang et du muscle du Rat en croissance. Ann. Biol. anim. Bioch. Biophys., 8, 517-530.

SAUVANT D., ROBERT J. C., 1983. IVe Symp. Int. Met. et Nutr, azotés, p. 105-109, Ed. I.N.R.A. publ. Les colloques, $n^{\circ} 16$.

SAUVANT D., ROBERT J.-C., 1986. Relation entre les teneurs en acides aminés libres du sang et la production de lait chez la chèvre en $4^{\mathrm{e}}$ semaine de lactation. Reprod. Nutr. Dévelop., 26. 343-344. 